\title{
LA ATENCIÓN TEMPRANA EN EL TRASTORNO DEL ESPECTRO AUTISTA: ESTADO DE LA CUESTIÓN Y DESAFÍOS PENDIENTES
}

\author{
EARLY CARE IN CHILDREN WITH AUTISM SPECTRUM \\ DISORDER: STATE OF THE ART AND REMAINING \\ CHALLENGES
}

\begin{abstract}
$\overline{\text { Rosabel Roig-Vila } a^{a^{*}} y \text { María E. Urrea-Solano }{ }^{a}}$
\end{abstract}
Fechas de recepción y aceptación: 28 de octubre de 2019 y 24 de marzo de 2020

DOI: https://doi.org/10.46583/edetania_2020.58.508

Resumen: El trastorno del espectro del autismo (TEA) constituye, actualmente, una de las alteraciones del desarrollo con mayores tasas de prevalencia. Sus elevadas cifras, unidas a las dificultades para la comunicación e interacción social, los patrones repetitivos y estereotipados de comportamiento y el repertorio limitado de intereses que presentan los menores con TEA, lo convierten, en la práctica, en una de las cuestiones más complejas para la sociedad $y$, en consecuencia, para los profesionales educativos. En este trabajo se abordan los principales hitos respecto a estas cuestiones, así como la consideración de la atención temprana (AT) como un elemento fundamental para hacer frente a este reto. En efecto, las técnicas de detección precoz e intervención interdisciplinar con el menor, la familia y el entorno, que ofrece la AT, representan una estrategia de reconocida eficacia. Sin embargo, los avances en la investigación y los cambios experimentados en la conceptualización del trastorno, junto con el impulso que supone el nuevo paradigma de AT, plantean la necesidad de avanzar en el conocimiento sobre los desafíos que aún quedan sin resolver en este ámbito.

Palabras clave: trastorno del espectro del autismo, atención temprana, primera infancia, intervención socioeducativa.

a Facultad de Educación. Universidad de Alicante.

${ }^{*}$ Correspondencia: Universidad de Alicante. Facultad de Educación. Calle Aeroplano s/n. 03690 (San Vicente del Raspeig,), Alicante. España.

E-mail: rosabel.roig@ua.es 
Abstract: The Autism Spectrum Disorder (ASD) currently stands out as one of the developmental disorders with higher prevalence rates. Its high figures, added to the difficulties for communication and social interaction, the repetitive and stereotyped behavior patterns, as well as the limited range of interests shown by minors diagnosed with ASD, actually turn it into one of the most complex issues faced by society and, accordingly, by educational professionals. This paper deals with the most important milestones in this regard, additionally focusing on the analysis of Early Care (EC) as a key element to cope with this challenge. As a matter of fact, the early detection techniques offered by EC -which include an interdisciplinary intervention with the minor, the family and the environment- have proved to be an effective strategy. However, both the research breakthroughs and the changes operated in the conceptualization of this disorder, along with the impetus given by the new EC paradigm, suggest a compelling need to make progress in the knowledge about the challenges which remain unsolved in this field text.

Keywords: autism spectrum disorder, early care, early childhood, socio-educational intervention.

\section{INTRODUCCIÓN}

El proceso de universalización de la enseñanza en la etapa comprendida entre los 0 y los 6 años, que está teniendo lugar en la actualidad, parece representar un auténtico desafío pedagógico para la sociedad y, por ende, para los sistemas educativos de la mayoría de los países (Creer, 2018). Las transformaciones acaecidas a nivel social, familiar y laboral durante la pasada centuria, junto con el desarrollo y consolidación de la filosofía inclusiva, han provocado la necesidad de arbitrar medidas para la atención socioeducativa de todos los niños y niñas desde su nacimiento. Por ello, más allá de los debates en torno a las posibles fortalezas y debilidades derivadas de una escolarización temprana (Narodowski y Snaider, 2017), lo cierto es que cada vez son más las organizaciones educativas que ofrecen servicios de atención y apoyo durante las primeras etapas, en un intento por promover la igualdad de oportunidades de los alumnos y alumnas con necesidades específicas de apoyo educativo (NEAE) (Romero-Galisteo, Morales-Sánchez y Hernández-Mendo, 2015). Además de focalizarse en el menor, la concepción contemporánea de este tipo de recursos insiste en la necesidad de incidir sobre la familia y el entorno, en el que este se desenvuelve, para fomentar el máximo desarrollo de sus capacidades y mejorar su calidad de vida y la de sus familiares. 
En este marco, uno de los colectivos que tradicionalmente parece haberse beneficiado de los efectos potenciales de los programas de atención temprana (AT) son los niños y niñas con trastorno del espectro del autismo (TEA) (Fuller y Kaiser, 2019). Actualmente, ningún profesional cuestiona el alcance que tiene la detección e intervención precoz para la mejora general del pronóstico, sobre todo cuando existe un elevado riesgo de que el desarrollo evolutivo ulterior pueda verse seriamente afectado, tal y como sucede en esta condición (Busquets et al., 2018). A través de estos equipos interdisciplinares, se interviene para facilitar la inclusión del menor con TEA en los distintos entornos en los que participa, respetando en todo momento su autonomía, personalidad y dignidad. Ahora bien, aunque la AT represente uno de los paradigmas de actuación más utilizados en este tipo de trastornos, también es uno de los que se precisa de investigación adicional, sobre todo teniendo en cuenta las posibilidades que presenta para mejorar el equilibrio socioemocional de la familia y el desarrollo de todas las áreas vitales del menor.

\section{AproximACIÓN TEÓRICA AL ESTUDIO DEL TEA}

\subsection{Una mirada hacia el pasado: evolución histórica del TEA}

En 1943 el fundador de la psiquiatría infantil y padre del autismo, Leo Kanner, publicó un estudio centrado en el análisis de la sintomatología de lo que él definió como trastorno autista del contacto afectivo (Nazeer, Hashemi, Imran y Azeem, 2019). En dicha publicación Kanner describía el comportamiento y el estilo de crianza recibido por un grupo de 11 niños y niñas, con edades comprendidas entre los 2 y los 8 años, cuya conducta se caracterizaba por la rigidez y la falta de flexibilidad, las dificultades para la interacción social y las limitaciones en la comunicación y el lenguaje. Aunque esta descripción que hizo Kanner sea considerada como la primera definición del autismo, el relato clínico primigenio fue obra de la soviética Grunya Sukhraeva en el año 1926 (Fitzgerald, 2019). En 1944 el también psiquiatra infantil Hans Asperger centró su atención en la alteración del contacto que presentaban estos niños, su particular manera de comunicarse, su portentosa capacidad cognitiva y memorística en determinadas áreas, las dificultades que mostraban para el 
ajuste social y los patrones de comportamiento estereotipado e irregular que presentaban. En su caso, Asperger optó por la denominación de psicopatía autística (Feinstein, 2019). No resulta extraño, sin embargo, que tanto Kanner como Asperger utilizasen el término autismo para referirse a este trastorno, ya que etimológicamente proviene del griego autos que significa "consigo mismo" (Evans, 2013).

Estas primeras descripciones dieron origen a la formulación de una serie de posibles explicaciones etiológicas durante los años cincuenta y sesenta, centradas preferentemente en la relación patológica de los progenitores con sus hijos e hijas (Harris, 2018). En general, se consideraba que estos menores, potencialmente neurotípicos, padecían un trastorno emocional severo como consecuencia de un estilo frío de crianza, situaciones de estrés muy intenso durante la primera infancia y/o la existencia de problemas en los progenitores. La falta de confirmación empírica de estas teorías, unida a la asociación del autismo con otros trastornos y a la falta de eficacia de la psicoterapia en el tratamiento, favorecieron la búsqueda de nuevos modelos explicativos (Wolff, 2004). Entre otras hipótesis, se insinuaron alteraciones en la capacidad de comprensión lingüística (Churchill, 1972) y un déficit simbólico global (Wing, 1972), propuestas que dejaban atrás las disfunciones afectivas y se centraban, prioritariamente, en las anomalías de carácter cognitivo.

Un avance importante producido durante la década de los ochenta será la ampliación del concepto, como consecuencia de la investigación epidemiológica de Wing y Gould (1979). Gracias a este estudio se identificó la denominada triada autista, a saber, la alteración de las habilidades comunicativas, el déficit en la interacción social y las dificultades en la imaginación, todo ello asociado a un patrón de comportamiento repetitivo y rígido. Será también en esta etapa cuando el síndrome pase a ser considerado como un trastorno del desarrollo, lo que planteará la necesidad de analizar, desde un punto de vista evolutivo, los posibles mecanismos o procesos que puedan verse afectados (Wolff, 2004). Entre otras posibles causas se sugirieron desequilibrios en la función ejecutiva (Damasio y Maurer, 1978), la ausencia o deterioro de la teoría de la mente (Baron-Cohen, Leslie y Frith, 1985) y las dificultades para la integración de la información (Frith, 1989); modelos teóricos que intentaron, con mayor o menor aceptación, arrojar luz sobre el posible origen del trastorno. 


\subsection{Definición del TEA, criterios diagnósticos y síntomas asociados}

En la actualidad el TEA se define como un trastorno del neurodesarrollo, caracterizado por las dificultades en la interacción social, la comunicación y la flexibilidad mental, que se manifiesta antes de los 30 primeros meses de vida, provocando un deterioro emocional y cognitivo de gravedad variable (Busquets et al., 2018). A pesar de la diversidad de manifestaciones clínicas, se puede afirmar, de manera general, que los rasgos distintivos del comportamiento de los niños y niñas con TEA incluyen un repertorio restrictivo de intereses y actividades, dificultades en la atribución de pensamientos, sentimientos y deseos en sus relaciones con los demás, así como deficiencias importantes en el área expresiva y comprensiva del lenguaje (Al Shirian y Al Dera, 2015).

La publicación de la última versión del Manual Diagnóstico de los Trastornos Mentales (DSM, por sus siglas en inglés) de la Asociación Americana de Psiquiatría (2013), el DSM-5, ha supuesto importantes cambios en la conceptualización del trastorno. En él desaparecen los distintos trastornos que formaban parte de los trastornos generalizados del desarrollo (trastorno autista, trastorno de Asperger, trastorno de Rett, trastorno desintegrativo infantil, autismo atípico y trastorno generalizado del desarrollo no especificado) para quedar todo englobado en una sola categoría diagnóstica de perspectiva dimensional llamada "trastorno del espectro del autismo" (Ezpeleta y Toro, 2014). Esta modificación en la denominación viene motivada, especialmente, por el deseo de enfatizar la multidimensionalidad del trastorno en las dos áreas que pueden verse afectadas (sociocomunicativa, e intereses fijos y conductas repetitivas). Además, entendida como un continuo, la noción de espectro se ajusta con más precisión a la variabilidad de manifestaciones clínicas (Doernberg y Hollander, 2016). De hecho, el reciente estudio de Wiggins et al. (2019) ha evidenciado que el empleo de estos nuevos criterios diagnósticos facilita y mejora notablemente el diagnóstico del TEA en menores con edades comprendidas entre los 2 y los 5 años.

La undécima versión de la Clasificación Estadística Internacional de Enfermedades y Problemas Relacionados con la Salud (CIE-11) (OMS, 2018), por su parte, ha incidido también en la actualización de los criterios para el diagnóstico y en la reformulación de las áreas afectadas. Además, deja de 
vincular el TEA con los trastornos mentales y lo clasifica dentro de las alteraciones del comportamiento y del neurodesarrollo. Otro cambio introducido por el CIE-11 ha sido la diferenciación entre los casos en los que se produce comorbilidad con otras condiciones y aquellos en los que el trastorno se manifiesta de manera exclusiva. En lo referente al diagnóstico infantil, resta importancia al tipo de juego que desarrollan los menores y se focaliza, preferentemente, en la posible vulneración e imposición de reglas mientras estos se hallan jugando. Con ello, se intenta mejorar la identificación y detección precoz de la condición, evitando el posible impacto de los valores culturales en los procesos diagnósticos (Angell, Empey y Zuckerman, 2018).

Respecto a la sintomatología y criterios del trastorno, el DSM-5 (Asociación Americana de Psiquiatría, 2013) establece que el sujeto debe presentar, en el momento presente o por sus antecedentes, los siguientes signos:

- Comunicación e interacción social (todos simultáneamente):

- Déficits persistentes en la reciprocidad socioemocional: pueden oscilar desde una interacción social anormal y dificultades para mantener una conversación en ambos sentidos, pasando por intereses, emociones y afectos restringidos, hasta la incapacidad absoluta de interacción social.

- Déficits en la conducta comunicativa no verbal para la interacción social: pueden comprehender desde un estilo comunicativo escasamente integrado, pasando por alteraciones en el contacto visual y en el lenguaje corporal o dificultades en el uso de la comunicación no verbal, hasta la ausencia total de expresiones y comunicación gestual.

- Deficiencias en el establecimiento, desarrollo y comprensión de relaciones: pueden incluir desde limitaciones en el ajuste del comportamiento al contexto, pasando por dificultades en el juego simbólico, hasta la falta total de interés por el resto de personas.

- Comportamiento, intereses y actividades (al menos dos):

- Habla, manipulación de objetos y movimientos de manera repetitiva y estereotipada (ecolalia, estereotipias motoras simples, frases idiosincráticas, etc.).

- Excesiva rigidez en las rutinas y patrones ritualizados de conducta verbal y no verbal (incomodidad ante pequeñas alteraciones, insistencia por comer la misma comida y seguir la misma ruta, etc.). 
- Intensidad desmesurada por intereses notoriamente restrictivos (fuerte apego hacia objetos inusuales, intereses excesivamente restringidos y repetitivos).

- Hiperreactividad o hiporreactividad ante estímulos sensoriales (indiferencia al dolor y/o a la temperatura, fascinación intensa por movimientos, luces u objetos del entorno, etc.).

- Los síntomas deben presentarse desde la primera infancia, aunque pueden no llegar a manifestarse por completo hasta que las demandas sociales no superen a las capacidades del sujeto.

- La sintomatología provoca un importante deterioro en el funcionamiento cotidiano de la persona.

- Este conjunto de síntomas no puede ser mejor explicado por discapacidad intelectual o un retraso global del desarrollo.

En función del grado de afectación de los criterios de comunicación social y de los intereses restringidos y conductas repetitivas, el DSM-5 (Asociación Americana de Psiquiatría, 2013) establece tres niveles de severidad, definidos por el tipo de apoyo que precisa el individuo para su adecuado desenvolvimiento diario: (1) requiere soporte, (2) urge de apoyo sustancial y (3) necesita de ayuda muy notable.

\subsection{Etiología y prevalencia}

A pesar de las múltiples investigaciones realizadas, por el momento no ha sido posible establecer una única causa capaz de explicar por sí misma la aparición del trastorno. Por ello, gran parte de la comunidad científica tiende a hablar más bien de un origen multifactorial y diverso, aunque con un importante peso del componente genético (Zeidán-Chuliá et al., 2013). La neurociencia cognitiva, por su parte, ha permitido poner de manifiesto la existencia de una estrecha relación entre el TEA y la etapa prenatal. De hecho, un reciente estudio llevado a cabo por un equipo de investigadores de la Universidad de Carolina del Norte viene a señalar que el trastorno puede estar causado por alteraciones en la conformación de la corteza cerebral (Nakagawa et al., 2019). Estas vendrían provocadas por una mutación en el gen Memo1, 
encargado de las estructuras en las que se organizan los progenitores radiales, responsables de la adecuada formación del cerebro.

Otros estudios, en cambio, inciden en la capacidad que los factores ambientales tienen para disparar los componentes biológicos, como por ejemplo la exposición de la madre durante el embarazo a ciertos productos químicos (Deng et al., 2015). Otras posibles causas que se han intentado vincular con el TEA son la edad de los progenitores (Wu et al., 2017), complicaciones durante el embarazo y el parto (Gardener, Spiegelman y Buka, 2011), enfermedades previas de la madre (Wang, Tang, Xu, Weng y Liu, 2016), la toma de medicamentos durante el embarazo (Gentile, 2014) y el consumo de tabaco por parte de la madre (Jung, Lee, McKee y Picciotto, 2017). Mención significativa merece la popularidad que está adquiriendo en los últimos años la hipótesis que vincula el autismo con la vacunación infantil, aunque esta parece carecer de respaldo empírico (Hvidd, Hansen, Frisch y Melbye, 2019). A la vista de tales resultados, todo parece apuntar a que se precisa de más investigaciones para poder identificar, de manera clara y concisa, la causa desencadenante del trastorno.

En cuanto a la prevalencia, los últimos estudios señalan que las cifras se sitúan en torno al 16,8 \% por cada 1.000 habitantes, es decir, un menor de cada 59 nacidos tendrá TEA (Baio et al., 2018), apreciándose un aumento significativo de los casos en más de dos puntos porcentuales durante los últimos años (Zablotsky et al., 2019). En España, no obstante, la manifestación del trastorno no parece alcanzar cifras tan elevadas. Por ejemplo, en Cataluña la prevalencia para el año 2017 fue del 1,23\%, con un incremento del 0,16\% desde el año 2009 (Pérez-Crespo et al., 2019). De igual manera, se ha subrayado la mayor incidencia del trastorno entre los varones, con una proporción de 4.5:1 (niños:niñas) (Pérez-Crespo et al., 2019). Se ha apreciado también que la sintomatología comienza a manifestarse, de manera variable, en torno a los 18 meses, precedida de un historial evolutivo aparentemente normal (Reynoso, Rangel y Melgar, 2019). Hasta esa edad, las evidencias son muy sutiles y en ocasiones pueden pasar desapercibidas, ya que están relacionadas, en la mayoría de los casos, con el déficit en los gestos protodeclarativos. Será a partir del segundo año de vida cuando las alteraciones evolutivas se manifiesten con mayor claridad. Por ello, tal y como afirman Brett, Warnell, 
McConachie y Parr (2016), reconocer los indicadores que pueden facilitar un diagnóstico e intervención tempranas representa una necesidad de primer orden.

\section{Conceptualización y desarrollo de LA AT}

El desarrollo evolutivo del menor constituye, ante todo, un proceso dinámico y complejo, en tanto que depende no solo de los aspectos biológicos, sino también de los factores sociales y culturales del contexto en que tiene lugar el nacimiento y desenvolvimiento posterior del sujeto. A ello se ha de añadir, además, que los primeros años de vida constituyen una etapa crítica, ya que en ellos se definen las habilidades de carácter motriz, cognitivo, lingüístico, social y afectivo que, posteriormente, permitirán al sujeto una adecuada interacción con el entorno que le rodea. En este escenario concreto emerge el paradigma de la AT, cuyo objetivo pasa por ofrecer a los niños y niñas, con algún tipo de trastorno del desarrollo o con posibilidad de padecerlo en un futuro, un conjunto de medidas y servicios que favorezcan su máximo potencial en todos los ámbitos y facetas de la vida (GAT, 2000).

Los modelos teóricos que la inspiran abarcan desde la teoría general de sistemas de Bronfenbrenner (1979), pasando por el enfoque transaccional del desarrollo de Sameroff y Chandler (1975) y el modelo de los sistemas evolutivos de Guralnick (2011), hasta la teoría transdisciplinar de King et al. (2009). Junto a ello debemos subrayar los aportes de algunas disciplinas como la neurología, la psicología, la fisioterapia, la pedagogía y la logopedia, áreas a las que pertenecen la mayoría de los especialistas que integran los equipos interdisciplinares de intervención. Fruto de la influencia de este marco analítico, la AT ha ido evolucionando y transitando desde un enfoque de actuación, centrado exclusivamente en el menor, hasta otro que hace cada vez más partícipe a la familia y al contexto donde el niño se desenvuelve. Su objetivo, por tanto, es ofrecer a los progenitores y al entorno circundante una serie de estrategias, recursos y orientaciones, como vía de empoderamiento, para un mejor funcionamiento familiar y del menor (Dunst y Trivette, 2009). 


\subsection{Los servicios de la AT: de la rehabilitación a la prevención}

La trascendencia de los primeros años de vida para el desarrollo físico, psicológico y emocional del menor, así como para la adquisición de las distintas habilidades sociales y personales, provocaron, a mediados de los años cincuenta del siglo xx, un especial interés por la primera infancia y por la aparición de posibles situaciones consideradas de alto riesgo para su salud. Esta preocupación, unida a la normativa internacional para la protección de la niñez, suscitó el surgimiento y el desarrollo posterior en EE. UU. de los programas de atención a la infancia, con objeto, inicialmente, de paliar los déficits que presentaban los niños y niñas en situación de riesgo de exclusión social (Guralnick y Bruder, 2019). En un principio, sin embargo, este tipo de prácticas presentó un carácter eminentemente rehabilitador, asistencial y compensatorio, sin contemplar las posibilidades de prevención que ofrece la AT y las consecuencias que esta tiene para la mejora del pronóstico.

En España sus orígenes se remontan a finales de la década de los sesenta, cuando un grupo de profesionales de Madrid y Barcelona comenzaron a trabajar con la idea de que una intervención temprana permite lograr mejores resultados; esto dio lugar, así, a los primeros servicios de estimulación precoz (Robles-Bello y Sánchez-Teruel, 2013). En 1973 tuvo lugar en Madrid el Curso breve teórico-práctico de estimulación precoz para niños menores de 5 años, considerado por Gutiez y Ruiz (2012) como el germen de la AT en nuestro país. También será destacable el impulso que supuso la promulgación en 1977 del Plan de acción de recuperación para minusválidos psíquicos del Servicio Estatal de Rehabilitación de Minusválidos (SEREM), así como las demandas de las familias afectadas, que empezaron a reclamar una mejor atención para sus hijos e hijas.

No será, sin embargo, hasta la década de los ochenta que, con el espaldarazo de la Ley 13/1982, de 7 de abril, de Integración Social del Minusválido (LISMI, 1982), se crearán en nuestro país los primeros servicios de estimulación precoz en los centros base de atención a la discapacidad (FEAPS, 2001). Esta norma introdujo un importante conjunto de medidas que venían a subrayar el alcance de la prevención y la necesidad de intervenir a una edad temprana (Giné, Balcells y Mas, 2010). A pesar de ello, los equipos de AT se 
siguieron caracterizando, sobre todo, por su enfoque clínico, ambulatorio y centrado en la rehabilitación de los déficits del menor y de la familia.

Durante los años noventa la AT pasará a ser abordada desde los servicios de salud, educación y servicios sociales, sin una clara distribución de las competencias y con marcadas diferencias entre las CC. AA. (Martínez y Calet, 2015). De hecho, mientras que Cataluña será la primera comunidad en atribuir la responsabilidad en esta materia al Departamento de Bienestar Social, Galicia será quien defina con mayor grado de detalle las prestaciones ofrecidas por este tipo de servicios.

Uno de los hitos más relevantes en su configuración, sin embargo, será la redacción del Libro Blanco sobre AT por parte de un grupo de profesionales de diversas CC. AA. y del Real Patronato de Prevención y Atención a las Personas con Minusvalía (GAT, 2000). Con él se pretendía establecer un marco de referencia y de intervención común para el conjunto de las administraciones y de los propios especialistas implicados. Supuso, además, el reconocimiento de la necesidad de ampliar la intervención a la familia y al entorno más amplio, en un intento por abandonar el carácter rehabilitador y clínico que hasta ese momento había caracterizado a la AT (Serrano, Mas, Cañadas y Giné, 2017).

En su proceso de consolidación también se debe destacar la publicación del Manual de buenas prácticas en el ámbito de la AT (FEAPS, 2001) y la elaboración del Plan de acción del CERMI estatal en materia de AT (CERMI, 2005). Más recientemente, y contemplando las recomendaciones internacionales en esta materia, se ha elaborado el Documento político del CERMI estatal sobre AT (CERMI, 2017), con el que se intenta evidenciar la necesidad de garantizar una AT de carácter universal, gratuita e inclusiva para todos los menores con trastorno o con riesgo de padecerlo. Asimismo, se ha de enfatizar la labor que está desarrollando en los últimos años Plena Inclusión (antigua FEAPS, Confederación Española de Organizaciones en favor de las Personas con Discapacidad) para alinear las prácticas de los centros de AT con el enfoque centrado en la familia (García-Grau, Martínez-Rico, McWilliam y Cañadas, 2019). 


\subsection{La práctica actual de la AT en España}

Fruto de la evolución experimentada durante los últimos cuarenta años, la AT se dirige hoy en día a los niños y niñas con edades comprendidas entre los 0 y los 6 años, que pueden presentar un alto riesgo de carácter biológico (nacimiento prematuro, bajo peso al nacer, etc.), de exclusión social (entornos con bajos niveles socioeconómicos, falta de cuidado, violencia doméstica, etc.) o con algún tipo de NEAE (Gutiez y Ruiz, 2012). Para la atención holística de sus necesidades, los centros de desarrollo infantil y atención temprana (CDIAT) o los centros de atención infantil temprana (CAIT), en función de la comunidad autónoma, están integrados por un equipo interdisciplinar de especialistas educativos, sanitarios y con formación en intervención social, lo que garantiza una actuación global en todas las áreas del sujeto (Juan-Vera y Pérez-López, 2009).

Si bien algunos de estos servicios están experimentando actualmente un proceso de transición hacia la adopción del modelo centrado en la familia, lo cierto es que son múltiples los estudios que vienen a poner de manifiesto la pervivencia del paradigma tradicional de AT, caracterizado, sobre todo, por el rol experto del profesional, el papel subsidiario de la familia y la intervención centrada en el menor (Gràcia et al., 2019; Serrano et al., 2017). A pesar de ello, las familias parecen mostrar un cierto grado de satisfacción con la atención recibida por este tipo de servicios, mostrando únicamente disconformidad con los elementos intangibles del proceso (Romero, Lillo y García, 2019). Los profesionales, por su parte, reclaman una mejor formación para ser capaces de implementar la perspectiva centrada en la familia, trabajar a partir de sus fortalezas, respetar sus decisiones y saber cómo responder ante las necesidades individuales de los padres y del entorno (Gràcia et al., 2019; Serrano et al., 2017). Parece, en consecuencia, que la adopción de este modelo sigue constituyendo un verdadero reto tanto para los especialistas como para las familias, con dificultades estas últimas para identificar sus puntos fuertes y lo que es importante para ellas.

El último informe sobre La realidad actual de la AT en España (GAT, 2013) también refleja un panorama de claroscuros, ya que, a pesar de la amplia red de servicios existentes en nuestro país, aún son múltiples los aspectos que se deben mejorar. En todo el territorio nacional únicamente catorce CC. AA. cuen- 
tan con una normativa reguladora para este tipo de servicios y en cuatro de ellas la intervención solo contempla hasta los 3 años. En cuanto a los centros, solo el 13 y el 4,83\% cuentan con actividades dirigidas de manera específica a la familia y al entorno respectivamente. En la mayoría de los casos, se trata de centros de titularidad pública $(48,14 \%)$, en los que trabajan entre tres y diez profesionales $(43,89 \%)$, especialmente logopedas $(18,12 \%)$, fisioterapeutas $(17,42 \%)$ y expertos en psicología $(15,89 \%)$, todos ellos preocupados sobre todo por la atención y la rehabilitación del menor $(65,88 \%)$. Se aprecian, además, serias limitaciones en cuanto a la naturaleza preventiva de este tipo de servicios, ya que, si bien la población atendida durante el año 2010 alcanzó los 43.986 menores, la intervención a la población que se encuentra en situación de riesgo dista mucho de ser una auténtica realidad.

\subsection{Los programas de AT en el TEA: desafíos pendientes}

Tal y como señalan Millá y Mulas (2009), la actuación temprana en el TEA presenta un carácter distintivo y específico que la diferencia de la intervención en otras alteraciones del desarrollo. Las variables que la distinguen se refieren, en primer lugar, a las peculiaridades del trastorno: la gran variabilidad de manifestaciones autísticas y la posible comorbilidad con otros síndromes. Asimismo, se ha de considerar el impacto que el trastorno genera en el entorno familiar, ya que el nacimiento de un hijo o hija con TEA suele provocar un nivel de estrés parental notoriamente severo, desequilibrios en la familia y necesidad de apoyo socioemocional (Lee y Chiang, 2018). Por ello, los programas de AT en el TEA no solo han de favorecer la adaptación del menor a su contexto vital, sino también capacitar a la familia para mejorar sus conocimientos y estrategias frente al trastorno. De hecho, se ha constatado que la formación dada a los padres de niños y niñas con TEA provoca notables mejoras comunicativas en los menores, reduce los síntomas depresivos de la madre y facilita la interacción en el núcleo familiar (Bradshaw, Steiner, Gengoux y Koegel, 2015).

Otro aspecto fundamental en este ámbito será el diagnóstico e identificación precoz, puesto que una detección temprana favorece una mejora notable del pronóstico y la evolución posterior del menor, pero también reduce los 
sentimientos de frustración, angustia e impotencia de los padres. En líneas generales, la edad de diagnóstico suele oscilar entre los 2 y 3 años y medio, dependiendo del contexto y de la heterogeneidad de los síntomas (Adelman y Kubiszyn, 2016), aunque hay algunos estudios que señalan que la identificación clínica de las primeras señales se puede producir en torno al primer año de vida (Busquets et al., 2018). Lo que parece quedar fuera de toda duda es la necesidad de mejorar la coordinación entre los diferentes servicios y recursos implicados en la atención a la infancia para identificar, de manera precoz, a los menores con posibilidades de presentar TEA (Boyd, Odom, Humprheys y Sam, 2010).

El proceso diagnóstico se ha de centrar, preferentemente, en la recogida de información referida a la historia evolutiva del menor, la aparición de los primeros signos, su comportamiento, su estilo comunicativo y de interacción, y el grado de participación que muestra en las situaciones grupales (Millá y Mulas, 2009). Recopilada esta información del entorno familiar y escolar, se ha de iniciar la exploración clínica por parte de un equipo interdisciplinar, con experiencia contrastada en el desarrollo de la primera infancia y con profundos conocimientos sobre el trastorno. De acuerdo con la literatura, las áreas que se deberán valorar son el comportamiento social, la comunicación y el lenguaje, así como el grado de rigidez mental que puede presentar el menor (Boyd et al., 2010). Para ello se habrán de utilizar pruebas específicas, pero también la observación de las conductas en su entorno natural.

En cuanto a la intervención, esta se ha de definir por la individualización y la flexibilidad, ya que el comportamiento de los menores con TEA suele experimentar una notable evolución en el tiempo, especialmente durante la primera infancia (Sánchez-Raya, Martínez-Gual, Moriana, Luque y Alós, 2015). Asimismo, en el diseño de los programas se ha de tener presente la singularidad y las necesidades de cada familia, puesto que la actuación no solo se ha de focalizar en la globalidad del niño o de la niña, sino también en la familia y en todos los contextos en los que el sujeto se desarrolla. Además, en aquellos casos en los que sea posible, será recomendable promover la participación e implicación del menor en la planificación de las actividades, partiendo en todo momento de sus intereses y necesidades.

La intervención, preferiblemente de carácter cognitivo-conductual, ha de garantizar la comprensión de las actividades por parte del menor y anticipar 
las tareas que se van a realizar en cada momento (Millá y Mulas, 2009). Las actividades, de carácter lúdico, funcional y significativo, han de partir de sus capacidades y potenciar sobre todo la participación en la dinámica social. Para ello, se podrán emplear apoyos técnicos, visuales, así como sistemas aumentativos y alternativos de comunicación. Sin embargo, el establecimiento de vínculos emocionales entre el equipo interdisciplinar y el menor será un requisito imprescindible.

En lo concerniente a la familia, será fundamental que esta sea considerada un objetivo y una aliada para la intervención, dados los efectos potenciales que tiene su participación e implicación activa para la mejora del menor (Bradshaw et al., 2015). Por tanto, los equipos de AT deberán hacer un esfuerzo empático por comprender la situación en la que se hallan los padres, ofrecerles un conjunto de herramientas y recursos apropiados, y apoyarles en todo el proceso. Respecto a la labor con el entorno, es muy importante facilitar el acceso y la máxima integración de la familia en las redes informales y en los servicios comunitarios, sean estos de carácter sanitario, social y/o escolar. En este último caso se habrá de procurar la necesaria colaboración con los profesionales del centro educativo para estimular el proceso de inclusión (Chiner, Gómez-Puerta y Cardona-Moltó, 2017) y el máximo desarrollo del niño en la escuela (García, Sánchez, Escorcia y Castellanos, 2012). Esta última usará un modelo basado en competencias (García-López, Gutiérrez, Pastor y Romo, 2018).

\section{A MODO DE CONCLUSIÓN}

A la vista de tal situación y repensando el futuro desarrollo de la AT respecto al TEA, se concluye señalando que, si bien han sido notables los avances logrados en este ámbito, también son múltiples los retos que restan por superar. En primera instancia, se estima conveniente mejorar la capacitación de los equipos interdisciplinares para poder lograr la plena aplicación y desarrollo del enfoque centrado en la familia y en el entorno. En segundo lugar, urge una mayor concienciación, implicación e inversión por parte de la administración educativa, ya que solo así se podrán ofrecer tiempos y espacios 
para la formación y colaboración profesional, así como para integrar las actuaciones en la escuela y la familia.

Por otra parte, es necesario homogeneizar la situación de la AT en todas las CC. AA. para lograr un panorama más uniforme y garantizar unos mínimos de cobertura a todos los niños y niñas con TEA. Una estrategia para ello puede ser la promulgación de un marco legislativo nacional que blinde el derecho universal a recibir una AT pública y gratuita para todas las familias. El fomento de la interdisciplinariedad y la transdisciplinariedad constituye, a su vez, un requisito básico para garantizar la eficacia y la calidad de la AT en el TEA, así como para favorecer la transformación de los equipos de intervención en agentes generadores de recursos, no solo para el propio menor, su familia y el entorno más cercano, sino también para el contexto social en el que se ubican. Así, la AT contribuirá decididamente a hacer realidad los derechos de los menores con TEA, de sus familias y de la sociedad.

\section{BibliografíA}

Adelman, C. R. y Kubiszyn, T. (2016). Factors that affect age of identification of children with autism spectrum disorder. Journal of Early Intervention 39(1), 18-32. DOI: https://doi.org/10.1177/1053815116675461.

Al Shirian, S. y Al Dera, H. (2015). Descriptive characteristics of children with autism at Autism Treatment Center, KSA. Physiology \& Behavior 151, 604-608. DOI: https://doi.org/10.1016/j.physbeh.2015.09.001.

Angell, A. M., Empey, A. y Zuckerman, K. (2018). A review of diagnosis and service disparities among children with autism from racial and ethnic minority groups in the United States. International Review of Research in Developmental Disabilities 55, 145-180. DOI: https://doi.org/10.1016/ bs.irrdd.2018.08.003.

Asociación Americana de Psiquiatría (2013). DSM-V Manual diagnóstico y estadístico de los trastornos mentales (5. ${ }^{\mathrm{a}}$ ed.). Madrid: Editorial Médica Panamericana.

Baio, J., Wiggins, L., Christensen, D. L., Maenner, M. J., Daniels, J., WarREN, Z.,... Dowling, N. F. (2018). Prevalence of autism spectrum disorder among children aged 8 years - autism and developmental disabilities 
monitoring network, 11 sites, United States, 2014. MMWR Surveillance Summaries 67(6), 1-23. DOI: 10.15585/mmwr.ss6706a1.

Baron-Cohen, S., Leslie, A. M. y Frith, U. (1985). Does the autistic child have a "theory of mind?". Cognition 21, 37-46.

Boyd, B. A., Odom, S. L., Humphreys, B. P. y SAm, A. M. (2010). Infants and toddlers with autism spectrum disorder: early identification and early intervention. Journal of Early Intervention 32(2), 75-98. DOI: https://doi. org/10.1177/1053815110362690.

Bradshaw, J., Steiner, A. M., Gengoux, G. y Koegel, L. K. (2015). Feasibility and effectiveness of very early intervention for infants at-risk for autism spectrum disorder: a systematic review. Journal of Autism and Developmental Disorders 45, 778-794. DOI: https://doi.org/10.1007/s10803-0142235-2.

Brett, D., Warnell, F., McConachie, H. y Parr, J. R. (2016). Factors affecting age at ASD diagnosis in UK: no evidence that diagnosis age has decreased between 2004 and 2014. Journal of Autism and Developmental Disorders 46(6), 1974-1984. DOI: https://doi.org/10.1007/s10803-0162716-6.

Bronfenbrenner, U. (1979). The ecology of human development. Cambridge: Harvard University Press.

Busquets, L., Mirabell, J., Muñoz, P., Muriel, N., Español, N., Viloca, L. y Mestres, M. (2018). Detección precoz del trastorno del espectro autista durante el primer año de vida en la consulta pediátrica. Pediatría Integral 22(2). Recuperado de: https://bit.ly/39hfK9q.

CERMI (2005). Plan de acción del CERMI estatal en materia de atención temprana a personas con discapacidad. Madrid: CERMI.

CERMI (2017). Documento político del CERMI estatal sobre atención temprana. Por el derecho primordial de la infancia a la salud y a su pleno desarrollo. Recuperado de: https://bit.ly/2JeqStc.

Chiner, E., Gómez-Puerta, M., y Cardona-Moltó, M. (2017). Internet and people with intellectual disability: an approach to caregivers' concerns, prevention strategies and training needs. Journal of New Approaches in Educational Research 6(2), 153-158. DOI: https://doi.org/10.7821/ naer.2017.7.243. 
Churchill, D. W. (1972). The relation of infantile autism and early childhood schizophrenia to developmental language disorders of childhood. Journal of Autism and Childhood Schizophrenia 2, 182-197.

Creer, A. (2018). Introducing everyday digital literacy practices into the classroom: an analysis of multi-layered media, modes and their affordances. Journal of New Approaches in Educational Research 7(2), 131-139. DOI: https://doi.org/10.7821/naer.2018.7.265.

Damasio, A. R. y Maurer, R. G. (1978). A neurological model for childhood autism. Archives of Neurology 35(12), 777-786.

Deng, W., Zou, X., Deng, H., Li, J., Tang, C., Wang, X. y Guo, X. (2015). The relationship among genetic heritability, environmental effects, and autism spectrum disorders: 37 pairs of ascertained twin study. Journal of Child Neurology 30(13), 1794-1799.

Doernberg, E. y Hollander, E. (2016). Neurodevelopmental disorders (ASD and ADHD): DSM-5, ICD-10, and ICD-11. CNS Spectrums 21(4), 295299. DOI: https://doi.org/10.1017/S1092852916000262.

Dunst, C. J. y Trivette, C. M. (2009). Capacity-building family-systems intervention practices. Journal of Family Social Work 12(2), 119-143. DOI: https://doi.org/10.1080/10522150802713322.

Evans, B. (2013). How autism became autism. The radical transformation of a central concept of a child development in Britain. History of the Human Sciences 26(3), 3-31. DOI: https://doi.org/10.1177/0952695113484320.

EzPeleta, L. y Toro, J. (coords.) (2014). Psicopatología del desarrollo. Madrid: Ediciones Pirámide.

FEAPS. (2001). Atención temprana para personas con retraso mental. Orientaciones para la calidad. Manuales de buena práctica. Madrid: FEAPS.

Feinstein, A. (2019). The history of autism education, en R. Jordan, J. M. Roberts y K. Hume (eds.), The Sage handbook of autism and education (pp. 24-42). Thousand Oaks: SAGE.

FitzGerald, M. M. (2019). The history of autism in the first half century of the $20^{\text {th }}$ century: new and revised. Journal of ReAttach Therapy and Developmental Diversities 1(2), 70-77. DOI: https://doi.org/10.26407/ 2018jrtdd.1.13.

Frith, U. (1989). Autism. Explaining the enigma. Oxford: Blackwell. 
Fuller, E. A. y KaIser, A. P. (2019). The effects of early intervention on social communication outcomes for children with autism spectrum disorder: a meta-analysis. Journal of Autism and Developmental Disorders. DOI: https://doi.org/10.1007/s10803-019-03927-z.

García, F. A., SÁnchez, M. ${ }^{a}$ C., Escorcia, C. T. y Castellanos, P. (2012). Valoración de la coordinación entre la atención temprana y educación infantil por educadores de escuelas infantiles. Edetania 41, 145-161.

García-Grau, P., Martínez-Rico, G., McWilliam, R. A. y Cañadas, M. (2019). Typical and ideal practices in early intervention in Spain during a transformation process of professional practices. Journal of Early Intervention. DOI: https://doi.org/10.1177/1053815119859046.

García-López, L., Gutiérrez, D., Pastor, J., y Romo, V. (2018). Validity and reliability of a questionnaire on primary and secondary school teachers' perception of teaching a competence-based curriculum model. Journal of New Approaches in Educational Research 7(1), 46-51. DOI: https://doi. org/10.7821/naer.2018.1.255.

Gardener, H., Spiegelman, D. y Buka, S. L. (2011). Perinatal and neonatal risk factors for autism: a comprehensive meta-analysis. Pediatrics 128(2), 344-355. DOI: https://doi.org/10.1542/peds.2010-1036.

GAT. (2000). Libro blanco de la atención temprana. Madrid: Real Patronato sobre Discapacidad.

GAT. (2013). La realidad actual de la atención temprana en España. Madrid: Real Patronato sobre Discapacidad.

GentiLe, S. (2014). Risks of neurobehavioral teratogenicity associated with prenatal exposure to valproate monotherapy: a systematic review with regulatory repercussions. CNS Spectrums 19(4), 305-315. DOI: https://doi. org/10.1017/S1092852913000990.

Giné, C., Balcells, A. y Mas, J. (2010). Early intervention in Catalonia. International Journal of Early Childhood Special Education 2(2), 97-110.

Gràcia, M., Simón, C., Salvador-Beltrán, F., Adam, A. L., Mas, J. M., Giné, C. y Dalmau, M. (2019). The transition process from center-based programmes to family-centered practices in Spain: a multiple case study. Early Child Development and Care. DOI: https://doi.org/10.1080/030044 30.2018 .1564916 . 
GURALNick, M. J. (2011). Why early intervention works: a systems perspective. Infants \& Young Children 24(1), 6-28. DOI: https://doi.org/10.1097/ IYC.0b013e3182002cfe.

Guralnick, M. J. y Bruder, M. B. (2019). Early intervention, en J. L. Matson (ed.), Handbook of intellectual disabilities. Integrating theory, research, and practice (pp. 717-741). Suiza: Springer.

Gutiez, P. y Ruiz, E. (2012). Orígenes y evolución de la atención temprana. Una perspectiva histórica de la génesis de la atención temprana en nuestro país. Agentes, contextos y procesos. Psicología educativa 18(2), 107-122. DOI: https://doi.org/10.5093/ed2012a12.

Harris, J. (2018). Leo Kanner and autism: a 75-year perspective. International Review of Psychiatry 30(1), 3-17. DOI: https://doi.org/10.1080/095402 61.2018.1455646.

Hvidd, A., Hansen, J., Frisch, M. y Melbye, M. (2019). Measles, mumps, rubella vaccination and autism: a nationwide cohort study. Annals of Internal Medicine 170, 513-520. DOI: https://doi.org/10.7326/M18-2101.

JuAn-Vera, M. ${ }^{a}$ J. y PÉRez-López, J. (2009). El funcionamiento de un centro de desarrollo infantil y atención temprana (CDIAT). Revista Interuniversitaria de Formación del Profesorado 23(2), 21-38.

Jung, Y., Lee, A. M., McKee, S. A. y Picciotto, M. R. (2017). Maternal smoking and autism spectrum disorder: meta-analysis with population smoking metrics as moderators. Scientific Reports 7(1), 4315-4325. DOI: https:// doi.org/10.1038/s41598-017-04413-1.

King, G., Strachan, D., Tucker, M., Duwyn, B., Desserud, S. y Shillington, M. (2009). The application of a transdisciplinary model for early intervention services. Infants \& Young Children 22(3), 211-223. DOI: 10.1097/ IYC.0b013e3181abe1c3.

Lee, J. K. y Chiang, H. M. (2018). Parenting stress in South Korean mothers of adolescent children with autism spectrum disorder. International Journal of Developmental Disabilities 64(2), 120-127. DOI: https://doi.org/10 .1080/20473869.2017.1279843.

LISMI (1982). Ley 13/1982, de 7 de abril, de Integración Social de los Minusválidos. Boletín Oficial del Estado, 30-04-1982. 
Martínez, A. y CALEt, N. (2015). Intervención en atención temprana: enfoque desde el ámbito familiar. Escritos de Psicología 8(2), 33-42. DOI: http://dx.doi.org/10.5231/psy.writ.2015.1905.

Millá, M. G. y Mulas, F. (2009). Atención temprana y programas de intervención específica en el trastorno del espectro autista. Revista de Neurología 48(2), 47-52.

Nakagawa, N., Plestant, C., Yabuno-Nakagawa, K., Li, J., Lee, J, Huang, C. W., ... Anton, E. S. (2019). Memo1-mediated tiling of radial glial cells facilitates cerebral cortical development. Neuron 103(5), 836-852. DOI: https://doi.org/10.1016/j.neuron.2019.05.049.

Narodowski, M. y Snaider, C. (2017). ¿Bebés en las escuelas? Infancias hiperescolarizadas en una cultura prefigurativa. Revista Latinoamericana de Ciencias Sociales, Niñez y Juventud 15(1), 45-57.

Nazeer, A., Hashemi, N., Imran, N. y Azeem, M. W. (2019). Autism spectrum disorder: a concept in evolution. Psychiatric Annals 49(3), 103-108. DOI: https://doi.org/10.3928/00485713-20190207-01.

OMS (2018). ICD-11. International classification of diseases $11^{\text {th }}$ revision. The global standard for diagnosis health information. Recuperado de: https://icd.who.int/en.

Pérez-Crespo, L. Prats-Uribe, A., Tobias, A., Duran-Tauleria, E., Coronado, R., Hervás, A. y Guxens, M. (2019). Temporal and geographical variability of prevalence and incidence of autism spectrum disorder diagnoses in children in Catalonia, Spain. Autism Research. DOI: https://doi. org/10.1002/aur.2172.

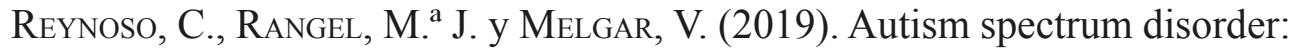
etiological, diagnostic and therapeutic aspects. Revista Médica del Instituto Mexicano del Seguro Social 55(2), 214-222.

Robles-Bello, M. ${ }^{a}$ A. y SÁnchez-Teruel, D. (2013). Atención infantil temprana en España. Papeles del Psicólogo 34(2), 132-143.

Romero, R. P., Lillo, M., y García, S. (2019). Calidad percibida en centros de atención temprana de Andalucía. Estudio cualitativo. Siglo Cero 50(2), 7-18.

Romero-Galisteo, R. R., Morales-Sánchez, V. y Hernández-Mendo, A. (2015). Desarrollo de una herramienta para la evaluación de la calidad per- 
cibida en los centros de atención infantil temprana. Anales de Psicología, 31(1), 127-136. DOI: <https://doi.org/10.6018/analesps.31.1.158191>.

Sameroff A. J. y Chandler, M. J. (1975). Reproductive risk and the continuum of caretaking causality, en F. D. Horowitz, E. M. Hetherington, S. Scarr-Salapatek y G. Siegel (eds.), Review of Child Development Research (pp. 187-244). Chicago: University of Chicago Press.

Sánchez-Raya, M. A., Martínez-Gual, E., Moriana, J. A., Luque, B. y Alós, F. (2015). La atención temprana en los trastornos del espectro autista (TEA). Psicología Educativa 21(1), 55-63. DOI: https://doi.org/10.1016/j. pse.2014.04.001.

Serrano, A. M., Mas, J. M., Cañadas, M. y Giné, C. (2017). Family systems and family centered intervention practices in Portugal and Spain. Iberian reflections on early childhood intervention, en H. Sukkar, C. J. Dunst y J. Kirkby (eds.), Early childhood intervention. Working with families of young children with special needs (pp. 177-196). Nueva York: Routledge. Wang, Y., TAng, S., Xu, S., Weng, S. y Liu, Z. (2016). Maternal body mass index and risk of autism spectrum disorders in offspring: a meta-analysis. Scientific Reports 30(6), 342-348. DOI: https://doi.org/10.1038/srep34248. Wiggins, L. D., Rice, C. E., Barger, B., Soke, G. N., Lee, L. C., Moody, E.... LEVy, S. E. (2019). DSM-5 criteria for autism spectrum disorder maximizes diagnostic sensitivity and specificity in preschool children. Social Psychiatry and Psychiatric Epidemiology 54(6), 693-701.

WING, L. (1972). Autistic children. Nueva York: Brunner.

WING, L. y GouLD, J. (1979). Severe impairments of social interaction and associated abnormalities in children: epidemiology and classification. Journal of Autism and Developmental Disorders 9(1), 11-29.

WolfF, S. (2004). History of autism. European Child \& Adolescent Psychiatry 13(4), 201-208. DOI: https://doi.org/10.1007/s00787-004-0363-5.

Wu, S., Wu, F., Ding, Y., Hou, J., Bi, J. y Zhang, Z. (2017). Advanced parental age and autism risk in children: a systematic review and meta-analysis. Acta Psychiatrica Scandinavica 135(1), 29-41. DOI: https://doi. org/10.1111/acps.12666.

Zablotsky, B., Black, L. I., Maenner, M. J., Schieve, L. A., Danielson, M. L., Bitsko, R. H.,... Boyle, C. A. (2019). Prevalence and trends of de- 
velopmental disabilities among children in the United States: 2009-2017. Pediatrics 144(4). DOI: https://doi.org/10.1542/peds.2019-0811.

Zeidán-Chuliá, F., Rybarczyk-Filho, J. L., Salmina, A. B., de Oliveira, B. H., Noda, M. y Moreira, J. C. (2013). Exploring the multifactorial nature of autism through computational systems biology: calcium and the rho GTPase RAC1 under the spotlight. NeuroMolecular Medicine 15(2), 364383. DOI: https://doi.org/10.1007/s12017-013-8224-3. 